\title{
The dynamic characteristics of polyurethane matrix of dye laser solid-state active elements
}

Ludmila F. Kosyanchuk ${ }^{\mathrm{a}}$, Nataly V. Kozak*a, Natalia V. Babkina ${ }^{\mathrm{a}}$, Volodymyr I. Bezrodnyy, Marina S. Stratilat ${ }^{\mathrm{a}}$

${ }^{\text {a }}$ Institute of Macromolecular Chemistry, National Academy of Sciences of Ukraine, Kharkov chaussee, 48, 02160 Kyiv, Ukraine

${ }^{\mathrm{b}}$ Institute of Physics, National Academy of Sciences of Ukraine, pr. of Science 46, 03680 Kyiv, Ukraine contactinge-mail kozaksmalt@ukr.net

Keywords: polyurethane, phenalenone dye, laser damage threshold, paramagnetic probe, radiation resistance.

Investigations of polyurethane matrices for solid-state laser active elements showed that comparison of chain mobility and permeability for low molecular substances of polyurethanes based on glycol and diisocyanate components of various structures allows predict their radiation resistance and choose optimal chemical composition of polyurethane. The correlation was shown of polyurethane dynamic properties with values of their elastic modulus and laser damage threshold under pulsed laser irradiation as well as the weak influence of phenalenone dyes introduction on the above characteristics.

\section{Introduction}

The necessary requirements imposed on polymeric matrices used in dye laser technology are as follows: the optical transparency in a wide spectral range, high solubility of the dye and its stability in polymer during storage and use. In addition, sufficient radiation resistance of polymer medium is of great importance for solid-state active laser element [1-3]. This option is highly dependent on the elasticity modulus of the polymer and its gas permeability. Decrease of elastic modulus and increase in gas permeability results in higher radiation resistance of the polymer due to prevention the material destruction caused by local heating under irradiation [1]. Elastomers and plasticized polymers have low modulus of elasticity, and hence increased radiation resistance. The improvement of the radiation resistance of widely used polymethyl methacrylate (PMMA) media is often achieved by its modifying with low molecular additives [4,5]. However such plasticization is accompanied by a deterioration of the mechanical properties and shape stability of PMMA based laser elements 
According to [6-8] polyurethane (PU) based elastic matrices have the high laser damage threshold. Varying PU components we can widely change the properties of the system. Therefore the comparison of chain mobility and permeability for low-molecular substances of polyurethanes of different structure allows predict the radiation resistance of various PU matrices in the solid state dye laser. In addition, it was interesting to study the effect of various phenalenone dyes on macrochain dynamic of dye doped PUs to choose optimal polyurethane matrix for the laser elements.

Dynamic characteristics of polyurethanes were studied by method of EPR using nitroxyl spin probe. Obtained EPR results were compared with the data of dynamic modulus of elasticity and measurements of the laser damage threshold.

\section{Experimental part}

\section{Materials}

Two-functional oligo-diethylene glycol adipate $\mathrm{MM}=800 \quad$ (ODA800); tri-functional oligo-oxypropylene glycol MM=500 (OPG-500) and two-functional oligo-oxypropylene glycol $\mathrm{MM}=1000$ (OPG-1000) were dried under vacuum at $90^{\circ} \mathrm{C}$ for $4 \mathrm{~h}$. Hexamethylene diisocyanate (HDI) and toluene diisocyanate (TDI) were distilled under vacuum. Trimethylolpropane (98\%) (TMP) was dried under vacuum at $40-45{ }^{0} \mathrm{C}$ for $2-4 \mathrm{~h}$. The 6amino-1-phenalenone (Ph-160), 2-chloro-6 (3- hydroxy propyl amino) fenalenon-1 (Ph-510) and 2-chloro- (3methyl amino) fenalenon-1 (Ph -439) (97\% of purity provided by Fluka) were used without purification. Dichloromethane $\left(\mathrm{CH}_{2} \mathrm{Cl}_{2}\right)$ has been distilled.

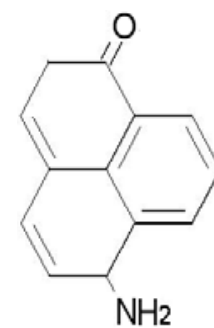

$P h-160$

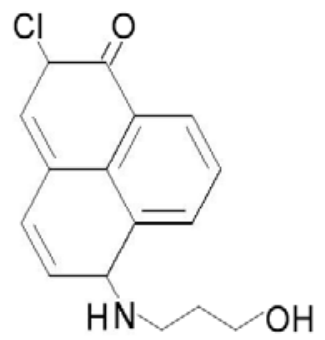

$P h-510$

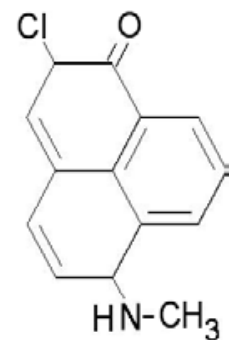

$P h-439$
Scheme 1. Phenalenone dyes structure.

\section{Synthesis}

The polyurethane matrix was prepared through the stage of prepolymer, based on HMDI or TDI and one of oligo-glycols: ODA800, OPG-500 or OPG-1000. At the next stage PU networks based on the prepolymer and trimethylolpropane $\mathrm{CH}_{3} \mathrm{CH}_{2} \mathrm{C}\left(\mathrm{CH}_{2} \mathrm{OH}\right)_{3}$ (TMP) as cross-linking agent were synthesized at $60^{\circ} \mathrm{C}$ during 10-12 h. The dye doped PU matrices were prepared by introduction of solution of F160 , $\mathrm{Ph}-510$ or $\mathrm{Ph}-439$ in $\mathrm{CH}_{2} \mathrm{Cl}_{2}$ into reaction mixture at the cross-linkage stage. It should be noted that F-160 can interact with components of the reaction mixture to form urea groups and Ph-510 can interact with components of the reaction mixture to form urethane groups [6]. Table 1 shows the compositions of the samples and their abbreviation. 
Table 1 The composition of the samples and their abbreviation

\begin{tabular}{|l|l|c|}
\hline \multicolumn{1}{|c|}{ Sample } & \multicolumn{1}{c|}{ Composition } & Dye \\
\hline PU-1 & OPG-500/HDI/TMP & - \\
\hline PU-1 (1) & OPG-500/HDI/TMP & Ph-160 \\
\hline PU-1 (2) & OPG-500/HDI/TMP & Ph-510 \\
\hline PU-1 (3) & OPG-500/HDI/TMP & Ph-439 \\
\hline PU-2 & ODA-800/HDI/TMP & - \\
\hline PU-2 (1) & ODA-800/HDI/TMP & Ph-160 \\
\hline PU-2 (2) & ODA-800/HDI/TMP & Ph-510 \\
\hline PU-2 (3) & ODA-800/HDI/TMP & Ph-439 \\
\hline PU-3 & OPG-1000/HDI/TMP & - \\
\hline PU-3 (1) & OPG-1000/HDI/TMP & Ph-160 \\
\hline PU-3 (2) & OPG-1000/HDI/TMP & Ph-510 \\
\hline PU-3 (3) & OPG-1000/HDI/TMP & Ph-439 \\
\hline PU-4 & OPG-1000/TDI/TMP & - \\
\hline PU-4 (1) & OPG-1000/TDI/TMP & Ph-160 \\
\hline PU-4 (2) & OPG-1000/TDI/TMP & Ph-510 \\
\hline PU-4 (3) & OPG-1000/TDI/TMP & Ph-439 \\
\hline
\end{tabular}

\section{Methods}

EPR-spectra were recorded at temperature $20^{\circ} \mathrm{C}$ using $3-\mathrm{cm}$ radio spectrometer $\mathrm{PE}-1306$ equipped with frequency meter ChZ-54. The magnetic field was calibrated using 2,2diphenil-1-pycrilhydrazyl (DPPH) $\quad(g=2,0036)$ and ions of $\mathrm{Mn}(2+)$ in $\mathrm{MgO}$ matrix ( $\mathrm{g}=2,0015)$.

Stable nitroxide radical 2,2,6,6tetramethylpiperidinyl-1-oxy (TEMPO) was used as paramagnetic spin probe. Into PU films nitroxide probe was introduced via diffusion from its saturated vapour at $30^{\circ} \mathrm{C}$ for 2 hours with subsequent keeping at $20^{\circ} \mathrm{C}$ for 24 hours [9]. Correlation time $(\tau)$ of the probe rotation in the range of its fast motion $\left(10^{-11}<\tau<10^{-9} \mathrm{~s}\right)$ was calculated for the matrices under investigation: by the known formula [10]:

$$
\left.\tau=6,65 \Delta H_{(+1)}\left(\sqrt{\left(I_{+1} / I_{-1}\right.}\right)-1\right) \times 10^{-10} C
$$

where $\mathrm{H}_{(1+)}$ - width of the spectra component in low field, in gauss; $I_{(+1)}, I_{(-1)}$ relative intensities of the spectra components in low and high field, respectively.

The dynamic elastic modulus (E') for PU films was evaluated by dynamic mechanical analysis (DMA) using the TA Instruments DMA Q800 equipment in the stretch mode. Measurements were made at temperatures from $298 \mathrm{~K}$ in the forced sinusoidal oscillation mode with a frequency of $10 \mathrm{~Hz}$.

Radiation strength of dye free polyurethane matrices was determined at the wavelength of the second harmonic $(532 \mathrm{~nm})$ of the single-mode Nd:YAG-laser MGL532 (Changchum New Industries Optoelectronics Tech. Co.) operating in single-pulse mode. The pulse generation duration $\tau_{i}$ was of 10 ns. Experimental scheme is presented at Figure 1.

The pulse laser damage threshold $E_{d}$ at 200 microns irradiation zone diameter was determined visually (with probability of 0.5 ). The damage was fixed using a tenfold magnifying glass.

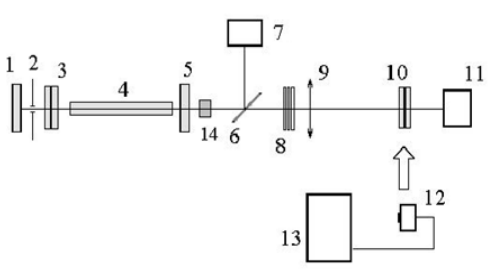

a

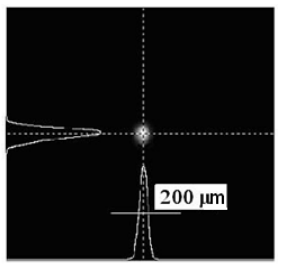

$b$
Figure. 1 Experimental scheme to determine polymer radiation resistance: (a) 1-5 laser, where: "blind" mirror (1); diaphragm (2); PLS (3); YAG (4); output mirror (5); 6 - beam splitter; 7, 11 - photodiodes; 8 - neutral filters; 9 - lens; 10 - the sample; 12 --CCD camera, oscilloscope; 13 - nonlinear crystal KTP; (b) - the beam cross section at the sample unit. 


\section{Results and discussion}

Integral intensity of EPR spectra is proportional to the amount of the TEMPO that is present in sample and it characterizes permeability of PU films for probe vapour. Paramagnetic probe correlation time $\tau$ characterizes the slowdown of its rotational diffusion in media. In polymer value of $\tau$ substantially depends on segmental mobility of macrochains. Increasing in macrochains mobility can go along with decrease of $\tau$ value.

Calculated values of $\tau$, the relative values of the integral intensity of EPR spectra $(I)$ with PU-1 system chosen as a comparison matrix, values of corresponding elasticity module $(E)$ and measured laser damage threshold of the PU $\left(E_{d}\right)$ are given in the Table 2.

Table 2. The parameters of the probe rotation and permeability in polyurethanes, elastic modulus and singlepulse laser damage threshold of the PUs

\begin{tabular}{|c|c|c|c|c|c|}
\hline Sample & $\begin{array}{c}\text { Densi } \\
\text { ty, } \\
\mathrm{kg} / \mathrm{m}^{3}\end{array}$ & $\begin{array}{c}\mathrm{I}, \\
\text { e.u }\end{array}$ & $\begin{array}{c}\tau \times 10^{9} \\
, \mathrm{c}\end{array}$ & $\begin{array}{c}\mathrm{E}^{\prime} \\
(298 \mathrm{~K}) \\
\mathrm{MPa}\end{array}$ & $\begin{array}{c}\mathrm{E}_{\mathrm{d}} \\
\mathrm{J} / \mathrm{cm}^{2} \\
( \pm 0,5)\end{array}$ \\
\hline PU-1 & 1,15 & 1,00 & 21 & 39.0 & 12,5 \\
\hline PU-1 (1) & 1,15 & 0,99 & 57 & 49,3 & - \\
\hline PU-1 (2) & 1,14 & 1,32 & 41 & 40,3 & - \\
\hline PU-1 (3) & 1,15 & 1,31 & 40 & 45,3 & - \\
\hline PU-2 & 1,27 & 5,6 & 5 & 5,4 & 18,0 \\
\hline PU-2 (1) & 1,24 & 7,1 & 6 & 5,5 & - \\
\hline PU-2 (2) & 1,21 & 8,3 & 5 & 5,6 & - \\
\hline PU-2 (3) & 1,20 & 5,2 & 4 & 5,4 & - \\
\hline PU-3 & 1,09 & 18,2 & 2 & 0,4 & 20,0 \\
\hline PU-3 (1) & 1,09 & 44,8 & 1 & 1,2 & - \\
\hline PU-3 (2) & 1,08 & 25,2 & 1 & 0,8 & - \\
\hline PU-3 (3) & 1,08 & 30,5 & 1 & 1,0 & - \\
\hline PU-4 & 1,12 & 8,5 & 2 & 2,7 & 13,5 \\
\hline PU-4 (1) & 1,13 & 5,9 & 3 & 3,1 & - \\
\hline PU-4 (2) & 1,13 & 5,5 & 3 & 3.0 & - \\
\hline PU-4 (3) & 1,13 & 9,3 & 3 & 4,1 & - \\
\hline
\end{tabular}

As can be seen from Table 2, for all PUs based on the aliphatic HMDI dynamic of macrochains characteristics, permeability of the matrix and its modulus are determined by glycol component structure. According to the data obtained PU-1 is characterized by the lowest mobility of the polymer chains, lowest permeability of probe from the gas phase and highest value of elastic modulus. That agrees with greatest degree of cross-linkage of PU-1 based on tri-functional OPG-500 of lowest oligoether molecular weight (MM) in the test series.

For PU-2 based on two-functional ODA800 the increase of macrochains mobility as well as probe permeability and decrease of elastic modulus value $E^{\prime}$ are observed (see Table 2) due to decreasing of glycol component functionality and due to increasing of this component MM. According to these parameters PU-2 occupies an intermediate position in the series: PU-1, PU-2, PU3. PU-3 based on two-functional OPG-1000 is characterized by highest values of chain mobility as well as probe permeability and by lowest value of elastic modulus in the series . Presence of ether groups in glycol component of PU-3 as compared with ester groups of PU-2 glycol component of is additional reason of higher flexibility of PU-3.

The determined values of radiation resistance of PU based on aliphatic HMDI agree well with the dynamic characteristics of $\mathrm{PU}$ matrices analyzed (Table 2) and their gas permeability. The best radiation resistance 
demonstrates PU-3 matrix. Rubber-like elasticity state of aliphatic polyurethane relieves the stresses arising under powerful laser pulse namely: local thermal effects and electrostriction caused mechanical stress. Increase in the gas permeability of the polymer also contributes to an increase in its radiation resistance. That is due to a pressure decrease in the heated region. In addition increase in the gas permeability also substantially reduces the so called "accumulation effect" of liquid and gaseous products of pyrolysis of the polymer under repeated laser pulses.

When changing the aliphatic diisocyanate component HMDI to aromatic TDI in a polyurethane matrix based on the same glycol (OPG-1000), we could expect the greater rigidity of PU-4 as compared with PU-3. The assumption of increasing the rigidity of the PU4 based on aromatic TDI is confirmed by increasing of probe correlation time value in PU-4 in comparison with PU-3. Furthermore, according to the data in Table 2 the ability TEMPO to diffuse in the PU-4 is essentially lower. Measured single-pulse laser damage threshold values also correlated with PU characteristics obtained using paramagnetic probe.

It should be noted that for all examined PU systems $E^{\prime}$ values determined from dynamic mechanical studies also correlate with EPR data (Fig. 2), in spite of the fundamental difference between the methods used.
As it can be seen from data in Table 2 the effect of phenalenone dyes of various structure on macrochain dynamic of dye doped PUs is relatively small. Nevertheless, it is reasonable to analyze effect of dye structure on these slight changes in more details.

In a series of PU-1 samples compact planar dye $\mathrm{Ph}-160$ [11] practically not change PU-1 (1) gas permeability whereas introduction of bulky dyes Ph- 510 and Ph-439 facilitates the probe permeability of PU-1 (2) and PU-1 (3) as compared with the original PU-1. The twofold increase of the correlation time values indicates suppression of polymer chains mobility in the presence of dyes. The most hindered probe rotation demonstrates PU-1 (1) where Ph-160 can form urea groups and strengthen hydrogen binding of glycol component. These results agree well with the increase in elastic modulus of dye doped PU-1 (Table 2).

In PU-2 series in the presence of dyes there is a tendency to lower $\tau$ values, practically unchanged elastic modulus value and evident change in probe permeability. The lowest probe mobility in PU-2 (2) in the presence of Ph-160 can be associated with strengthening of $\mathrm{H}$ bonding of urea groups with glycol component [11], and the highest permeability of PU-2 (2) can be associated with the presence of bulky nonplanar Ph-510 dye [9] linked with PU chain.

Almost the same results were observed for samples of the series on PU-3. Slight changes are observed in the polymer chains mobility and 
elastic modulus. Loosening of PU-3 in the presence of dyes may be due to the fact that large, bulky molecules of dye prevent compact arrangement of macrochains.

In PU-4 matrices containing aromatic fragments the presence of dyes results in reduced macrochains mobility. That can be associated with $\pi-\pi$ interaction of dyes condensed ring with aromatic diisocyanate component, which significantly stabilizes the molecular structure [12] reducing its mobility. The data correlate with an increase in elastic modulus (Table 2). Chemical binding of Ph-160 and Ph-510 dyes in PU-4 (1) and in PU-4 (2), respectively, leads to a compaction of the PU matrix and reduces its probe permeability. PU4(3) doped with chemically inert Ph-439 dye have probe permeability approximately the same as the original PU-4.

\section{Conclusions}

To summarize, the carried out investigations of polyurethane matrices for solid-state laser active elements showed that comparison of chain mobility and permeability for low molecular substances of polyurethanes based on glycol and diisocyanate of various structure allows predict their radiation resistance and choose optimal chemical composition of polyurethane.

According to obtained results the radiation resistance of phenalenone dyes doped polyurethane is primarily determined by the chemical structure of the polyurethane matrix. Changes in dynamic properties and elastic modules of the polyurethane in the presence of the dye are in most cases a trend. They are caused both by steric factors and by possibility of chemical interaction of the dye with polyurethane.

\section{References}

[1] Zemskyi VA, Kolesnikov YuL, Meshkovskyi IK. Fizika i tekchnica impulsnykh lazerov na krasitelyakh (Physics and technology of pulsed dye lasers). SPB SPbGU ITMO 2005;:176.

[2] Ishchenko A, Grabchuk G. Physical and chemical problems of the creation of photostable converters of light energy on the basis of dyed polymers. Theoretical and Experimental Chemistry 2009;45(3):143167. doi: 10.1007/s11237-009-9078-5

[3] Duarte F. Solid-state multiple-prism grating dye-laser oscillators. Appl. Opt. 1994;33(18):3857. doi: 10.1364/ao.33.003857

[4] Gromov D, Dyumaev K, Manenkov A, Maslyukov A, Matyushin G, Nechitailo V, Prokhorov A. Efficient plastic-host dye lasers. Journal of the Optical Society of America B 1985;2(7):1028. doi: 10.1364/josab.2.001028

[5] Maslyukov A, Sokolov S, Kaivola M, Nyholm K, Popov S. Solid-state dye laser with modified poly(methyl methacrylate)-doped active elements. Appl. Opt. 1995;34(9):1516. doi: 10.1364/ao.34.001516

[6] Kosyanchuk L, Bezrodna T, Stratilat M, Menzheres G, Kozak N, Todosiichuk T. Peculiarities of interactions between 6-aminophenalenone dye and polyurethane matrix. Journal of Polymer Research 2014;21(10):1-7. doi: 10.1007/s10965-014-0564-7

[7] Bezrodnyi V, Negryiko A, Klishevich G, Stratilat M, Kosyanchuk L, Todosiichuk T. Investigations of photophysical and generation properties of active 
elements based on dyes in aliphatic polyurethane matrix.

Journal of Polymer Research 2013;20(9):1-8. doi: 10.1007/s10965-013-0246-x

[8] Bezrodnyi V, Ishchenko A. Laser media based on coloured polyurethane. Quantum Electronics 2000;30(12):1043-1048. doi: 10.1070/qe2000v030n12abeh001862

[9] Kosyanchuk L, Stratilat M, Kozak N, Todosiichuk $\mathrm{T}$. The influence of the phenalenone dyes on the kinetics of the polyurethanes formation with different nature of the diisocyanate unit. Polim. J. 2015;37(3):235240.

[10] Kovarskii AL. Spin probes and labels. A quarter of a century of application to polymer studies, In: Pethrick R.A. editor. Polymer Yearbook 1996;13:113-139.

[11] Kosyanchuk L, Stratilat M, Kozak N, Todosiichuk T. Influence of diisocyanate component nature of polyurethanes doped with phenalenone dyes on rotational dynamics of nitroxyl paramagnetic probe. Ukr Chem J 2015;81(1):56-61.

[12] Luo R, Gilson H, Potter M, Gilson M. The Physical Basis of Nucleic Acid Base Stacking in Water. Biophysical Journal 2001;80(1):140-148. doi: 10.1016/s0006-3495(01)76001-8 